\section{(2) OPEN ACCESS}

\title{
Best supportive care and prognosis: advanced gastroesophageal adenocarcinoma
}

\author{
Kirsty E Cavanagh, ${ }^{1}$ Mark A Baxter (D) ${ }^{2,3}$ Russell D Petty ${ }^{2,3}$
}

\begin{abstract}
${ }^{1}$ School of Medicine, Ninewells Hospital and Medical School, University of Dundee, Dundee, UK

${ }^{2}$ Division of Molecular and Clinical Medicine, Ninewells Hospital and Medical School, University of Dundee, Dundee, UK

${ }^{3}$ Tayside Cancer Centre, Ninewells Hospital and Medical School, NHS Tayside, Dundee, UK
\end{abstract}

\section{Correspondence to} Dr Mark A Baxter, Division of Molecular and Clinical Medicine, University of Dundee School of Medicine, Dundee DD2 1SY, UK; m.z.baxter@dundee.ac.uk

Received 12 August 2020 Accepted 30 June 2021

\section{Check for updates}

(C) Author(s) (or their employer(s)) 2021. Re-use permitted under CC BY-NC. No commercial re-use. See rights and permissions. Published by BMJ.

To cite: Cavanagh $\mathrm{KE}$, Baxter MA, Petty RD. BMJ Supportive \& Palliative

Care Epub ahead of

print: [please include Day

Month Year]. doi:10.1136/

bmjspcare-2020-002637

\section{ABSTRACT}

Objectives Real-world data are lacking on survival in patients with advanced gastroesophageal adenocarcinoma (GOA) treated with best supportive care (BSC) alone. This knowledge is vital to personalise cancer treatment and obtain informed consent. This study aimed to define and compare survival in patients with advanced GOA treated with and without palliative chemotherapy (CTx), and to explore the factors that impact prognosis.

Methods Patients in NHS Tayside, Scotland, diagnosed with advanced GOA (defined as nonresectable) over a 2-year period were identified retrospectively. Clinical data were obtained from electronic records. Kaplan-Meier and Cox regression analysis were performed to determine median overall survival (mOS) and investigate contributing factors.

Results 127 eligible patients were identified. There was a significant difference in mOS between patients in the BSC and CTx groups (3.1 months vs 8.9 months, $p=0.00089$ ). This was maintained when those deemed not fit for CTx were removed. One-year survival was 16\% versus $33 \%$. Cox regression analysis in the BSC group identified stage $(p<0.001)$ and Eastern Cooperative Oncology Group performance status (ECOG PS) ( $p=0.013$ ) as having independent predictive value for survival. Age was not related to outcome. Palliative stents were inserted in 48 patients (37.8\%).

Conclusions To our knowledge, this is the largest reported study in Europe of outcomes in patients with advanced GOA treated with BSC only. The mOS with BSC is approximately 3 months. Cancer stage and ECOG PS have a role in prognostication at diagnosis. Our findings support the benefit of palliative chemotherapy in this population, and real-world survival corresponds to published trial data.

\section{INTRODUCTION}

The incidence of gastroesophageal adenocarcinoma (GOA) has risen steadily in the developed world over the last 40 years, primarily due to increased prevalence of obesity and gastroesophageal reflux disease. ${ }^{1}$ In the UK, there are approximately 15000 new cases annually, with almost half occurring in patients aged 75 years and over. ${ }^{2}$ Sixty to eighty per cent of patients present at an advanced stage with inoperable or metastatic disease, often following a period of reduced oral intake and significant symptom burden. ${ }^{3}$

There are limited treatment options in advanced GOA. Chemotherapy is the backbone of palliative therapy and for those patients fit and able to receive systemic therapy, median overall survival (mOS) in clinical trials is less than a year. ${ }^{4}$ In addition, palliative chemotherapy often has a high toxicity burden and impact on quality of life (QoL), with over half of patients requiring a hospital admission at least once during treatment. ${ }^{45}$

It is recognised that there is a mismatch in terms of age, fitness and comorbidity between real-world patients and those recruited to clinical trials on which survival and toxicity figures are based. For example, the median age in the Randomized ECF for Advanced and Locally Advanced Esophagogastric Cancer 2 (REAL-2) trial, which established a standard of care, was 63 compared with the median age of diagnosis of 74 years old in the population as a whole. ${ }^{4}$ We can therefore assume that the true impact of treatment on patient's QoL is likely to be greater.

This led to the Alternative chemotherapy for frail or elderly patients with advanced gastric or oesophageal cancer (GO2) trial, which investigated reduced dose chemotherapy in patients with advanced gastro-oesophageal cancer in whom there was a doubt over suitability for full-dose therapy. Reduced dose chemotherapy was found to be 
non-inferior in terms of survival (mOS was 7.6 months) and was better tolerated. ${ }^{6}$

Despite a wealth of data on survival outcomes with systemic therapy, very little data exist on survival with best supportive care (BSC) alone. BSC is defined as no further active anticancer treatment with a focus on symptom control. This can either be in the community or in a hospital/hospice setting. Most of the data that exist are historic and based on small patient numbers with mOS ranging from 3 to 5 months. ${ }^{7-9}$ Recent data from the BSC arm of the GO2 trial reported an mOS of 3.0 months. ${ }^{10}$ The data, however, do not capture those patients who are diagnosed in the community or hospital and never meet a cancer specialist.

Given the modest survival benefit provided by chemotherapy, to adequately inform patients regarding the risks and benefits of treatment and to guide clinicians, a better understanding of outcomes with supportive care only is required. This study aimed to address this problem. We sought to define and compare survival in patients with advanced GOA treated with and without palliative chemotherapy in our region, and to explore the baseline factors that may impact prognosis in those who do not receive systemic therapy.

\section{METHODS}

This was a retrospective study. Patients in NHS Tayside, Scotland, diagnosed with advanced GOA (defined as non-resectable) over a 2 -year period between 1 January 2016 and 31 December 2017 were identified using multidisciplinary team meeting records. Clinical data were then obtained from paper and electronic medical records. Those with pathological confirmation of oesophageal, gastroesophageal junctional or gastric adenocarcinoma were included. For all patients, baseline demographic data (age, sex, site of primary, stage, Eastern Cooperative Oncology Group performance status (PS)) were recorded. For those who were not seen in an oncology clinic, PS was obtained from the available medical records. The date of biopsy was taken as the date of diagnosis for all patients.

Survival was defined as date of diagnosis to date of death to enable comparison between groups with a censor date of 1 January 2020. Patients who either chose not to have active treatment with either systemic therapy or radiotherapy or were deemed not suitable were labelled 'best supportive care'.

Kaplan-Meier analysis for survival was performed to determine mOS using $\mathrm{R}$ statistical software version 4.0.2. Cox regression analysis was used to determine contributing baseline factors.

\section{RESULTS}

One hundred and fifty-two patients were diagnosed with advanced GOA in NHS Tayside during the time period. The median age at diagnosis was 73 (range $36-95)$ years. Fifty-two (34.2\%) of patients received palliative chemotherapy and $75(49.3 \%)$ had BSC
Table 1 Baseline demographic features of the patients with advanced gastroesophageal adenocarcinoma

\begin{tabular}{|c|c|c|c|}
\hline & $\begin{array}{l}\text { Best supportive } \\
\text { care (BSC) } \\
\end{array}$ & $\begin{array}{l}\text { Palliative } \\
\text { chemotherapy } \\
\end{array}$ & \\
\hline & $\mathrm{n}=75$ & $\mathrm{n}=52$ & $P$ value \\
\hline $\begin{array}{l}\text { Age (median, } \\
\text { years } \pm \text { range) }\end{array}$ & 80 (54-95) & $68(36-83)$ & 0.019 \\
\hline Sex & & & 0.56 \\
\hline Male & $50(67 \%)$ & $38(73 \%)$ & \\
\hline Female & $25(33 \%)$ & $14(27 \%)$ & \\
\hline ECOG PS & & & $<0.0001$ \\
\hline $0 / 1$ & $30(40 \%)$ & $44(85 \%)$ & \\
\hline 2 & $22(29 \%)$ & $5(10 \%)$ & \\
\hline$>2$ & $22(29 \%)$ & $1(2 \%)$ & \\
\hline Unknown & $1(1 \%)$ & $2(4 \%)$ & \\
\hline \multicolumn{4}{|l|}{ TNM stage } \\
\hline Stage $1 / 2$ & $16(21 \%)$ & $0(0 \%)$ & 0.0004 \\
\hline Stage 3 & $16(21 \%)$ & $9(17 \%)$ & \\
\hline Stage $4 a$ & $10(13 \%)$ & $6(12 \%)$ & \\
\hline Stage $4 b$ & $29(39 \%)$ & $37(71 \%)$ & \\
\hline Unknown & $4(5 \%)$ & $0(0 \%)$ & \\
\hline Stent inserted & $28(37 \%)$ & $20(38 \%)$ & 0.72 \\
\hline Albumin (g/dL) & $32(25-36)$ & 37 (32-39) & 0.034 \\
\hline \multicolumn{4}{|c|}{ Chemotherapy regime } \\
\hline EOX & & $28(54 \%)$ & \\
\hline OX & & $14(27 \%)$ & \\
\hline EOF & & $1(2 \%)$ & \\
\hline $\mathrm{ECX}$ & & $1(2 \%)$ & \\
\hline$C X$ & & $2(4 \%)$ & \\
\hline $\mathrm{CX}-\mathrm{H}$ & & $6(12 \%)$ & \\
\hline
\end{tabular}

The TNM Classification of Malignant Tumours (TNM) is a globally recognised standard for classifying the extent of spread of cancer. For this study, TNM edition 8 was used.

CX, cisplatin/capecitabine; CX-H, cisplatin/capecitabine/trastuzumab; ECOG PS, Eastern Cooperative Oncology Group Performance Status; ECX, epirubicin/cisplatin/capecitabine; EOF, epirubicin/oxaliplatin/5fluorouracil; EOX, epirubicin/oxaliplatin/capecitabine; OX, oxaliplatin/ capecitabine.

only. Twenty-five (16.4\%) patients had palliative radiotherapy only and were excluded from the analysis. Forty-eight (37.8\%) patients within the cohort required a palliative oesophageal/gastro-oesophageal junctional stent for dysphagia.

The patient demographics are shown in table 1. Of the 127 patients eligible for analysis, 39 (31\%) were women and $88(69 \%)$ men. Seventy-four (57\%) had a PS of 0 or $1,21 \%$ had a PS of 2 and $18 \%$ had a PS $>2$. Those who received chemotherapy were significantly younger than those who did not (median 68 vs 80 years; $\mathrm{p}<0.019)$, had more advanced disease $(\mathrm{p}=0.0004)$, were of better PS $(\mathrm{p}<0.0001)$ and had higher baseline albumin $(p=0.034)$. The most common palliative chemotherapy regime used was epirubicin/oxaliplatin/ capecitabine (EOX) (table 1).

There was a significant difference in mOS between patients in the BSC and CTx groups (3.1 months vs 


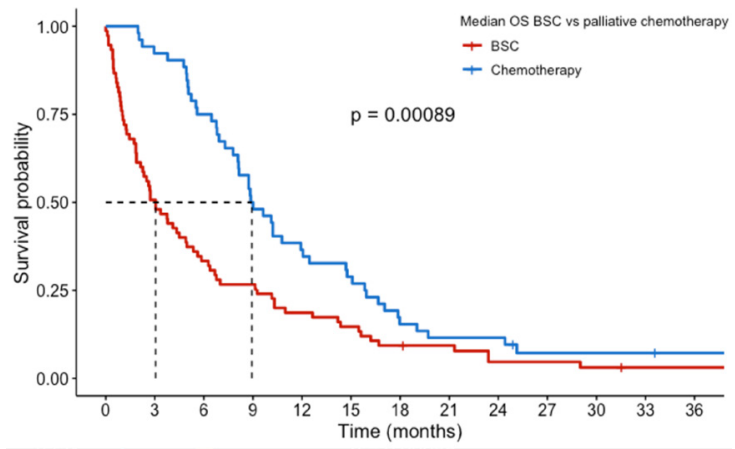

Figure 1 Kaplan-Meier survival analysis for NHS Tayside patients with advanced gastroesophageal cancer treated with palliative chemotherapy (blue line) or supportive care alone (red line). BSC, best supportive care; OS, overall survival.

8.9 months, $\mathrm{p}=0.00089$ ) (figure 1 ). This was maintained when those deemed not fit (defined as PS >2) for CTx were removed from the BSC cohort (3.9 months vs 8.9 months, $\mathrm{p}=0.012$ ). One-year survival was $16 \%$ in the BSC cohort and 33\% in those who received CTx. Patients who received CTx with PS 0,1 and 2 had mOS of 11.3 versus 8.1 versus 5.8 months, respectively.

Of the 52 patients who received first line palliative CTx, 16 (30.8\%) went on to receive second line systemic therapy and only $3(5.8 \%)$ received subsequent line therapy. Seven $(13.5 \%)$ patients died within 30 days of their last cycle of CTx and 27 (51.9\%) within 90 days.

Cox regression analysis in the BSC group identified increasing stage and poorer PS as having independent predictive value for survival (figure 2). An albumin
$<30 \mathrm{~g} / \mathrm{dL}$ at diagnosis was also associated with poorer survival. Older age or sex was not related to outcome. For those receiving CTx, only an albumin $<30 \mathrm{~g} / \mathrm{dL}$ was associated with poorer survival.

\section{DISCUSSION}

Advanced gastroesophageal adenocarcinoma is an increasingly common disease with a very poor prognosis. It is associated with high symptom burden and use of healthcare resources demonstrated by the significant use of out of hours services in the last year of life $^{11}$ and the requirement for stent insertion by over a third of patients shown by this study.

Chemotherapy can improve survival but with an impact on QoL due to treatment-related side effects as well as the need to attend hospital for treatment. Despite multiple clinical trials comparing treatment regimes, information is lacking as to prognosis without chemotherapy. This is essential to adequately guide both patients and clinicians in their decision making around palliative treatment, particularly in the context of recent reports in the UK and the USA highlighting that older patients with cancer often value QoL over length of life. ${ }^{12} 13$

In this study, we present real-world data from our cohort of patients in Scotland. The median age of diagnosis ( 73 years old) of our population and the male to female ratio of approximately $3: 1$ is in keeping with UK and Scottish national figures, ${ }^{2}{ }^{14}$ suggesting that this is a representative cohort.

We demonstrate that the median survival, in our real-world population, with supportive care alone is 96 days - in keeping with previously published data

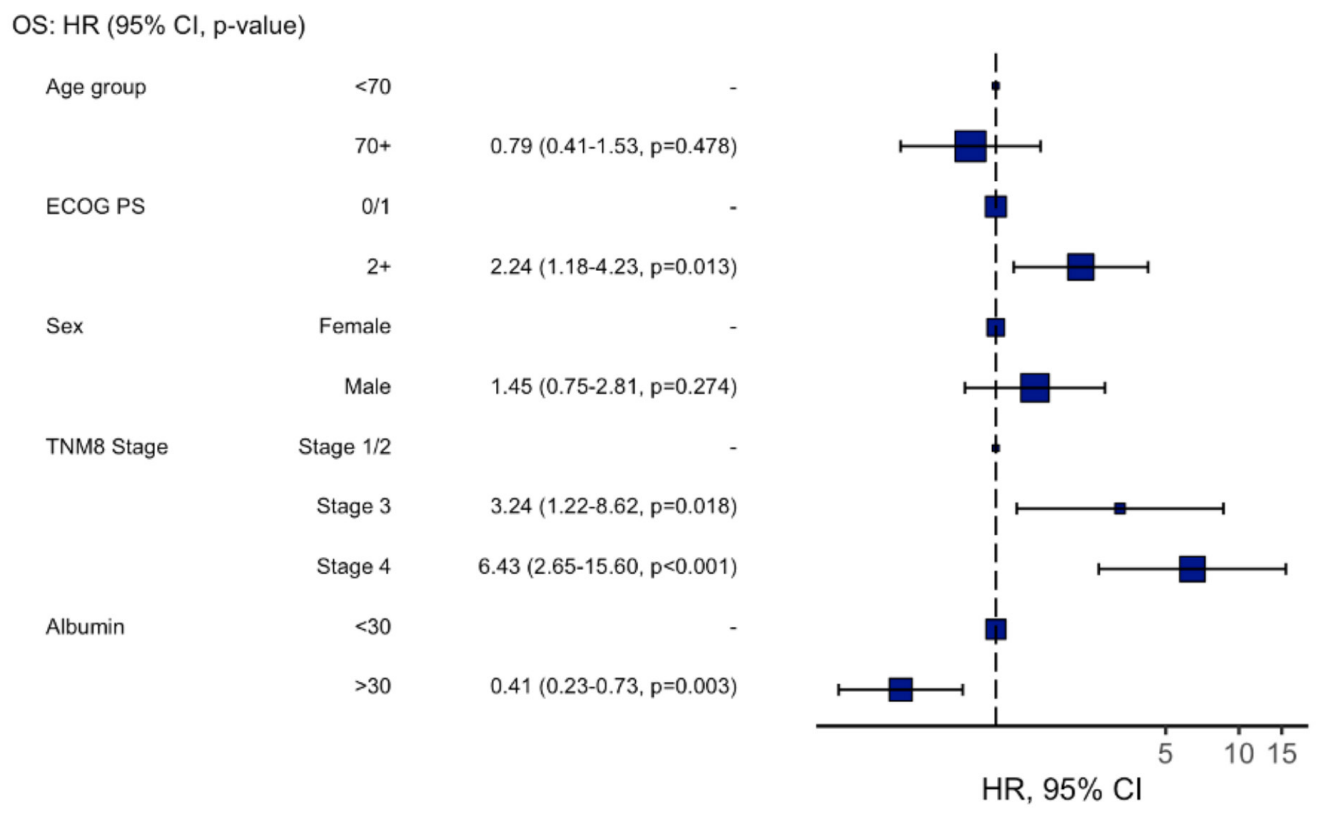

Figure 2 Cox regressional analysis for factors influencing survival in patients with advanced gastroesophageal adenocarcinoma treated with supportive care only. ECOG PS, Eastern Cooperative Oncology Group Performance Status; OS, overall survival; Tumour Node Metastasis classification; TNM. 
from randomised trials in smaller cohorts. ${ }^{7-10}$ We also demonstrate that stage of disease, PS and nutritional status (using the surrogate marker of albumin) have independent prognostic value in the context of BSC.

Age was not related to survival suggesting disease biology/stage and patient fitness are the main drivers in outcome. This is supported by the fact that almost one in five patients survived to 1 year without treatment, highlighting the range of disease trajectories. Despite this, those in the BSC arm were significantly older than those who received chemotherapy, likely reflecting a combination of patient choice, and an increased prevalence of frailty with age which is known to impact clinician confidence in prescribing treatment.

In those eligible for chemotherapy, defined as having a PS of $\leq 2$, survival was improved with treatmentmOS was approximately 8.9 months, an increase of 5.9 months compared with BSC. This benefit is similar to published data. ${ }^{15}$ The EOX arm of the REAL-2 trial, on which our standard of care is based, reported an mOS of 11.2 months-this is broadly similar to our cohort of patients with PS 0 but is greater than that in those with poorer PS. ${ }^{4}$ This again highlights the difference between trial and real-world populations and the importance of patient selection. It is also important to highlight that following progression on treatment, prognosis is poor. In our study $52 \%$ of patients died within 90 days of their last cycle of treatment.

Although QoL and symptom data were missing from our analysis, chemotherapy has been shown to improve symptom burden with subsequent QoL benefits in the palliative setting. ${ }^{7}$ The requirement of gastroesophageal stent insertion for more than a third of patients highlights the importance and demand for this procedure in the palliation of symptoms.

In summary, our data indicate that prognosis in advanced GOA is approximately 3 months with supportive measures, but in selected patients survival can be improved with chemotherapy. Our results provide real-world data for clinicians to reference when considering and discussing treatment options with patients, which will be invaluable for aiding prognostication. This is not only useful for cancer specialists but also the patient's primary care provider as we try to minimise harm from treatment toxicity and hospital admission. From a patient perspective, knowledge of their likely prognosis is an essential component of shared decision making, particularly when the burden of treatment is significant.

\section{CONCLUSION}

In advanced GOA, mOS with BSC alone is approximately 3 months, however, longer-term survival is seen. Treatment with chemotherapy improves survival in those who are fit to receive it. Knowledge of the benefit chemotherapy brings is an important factor in enabling patients to make an informed decision around their management.
Twitter Mark A Baxter@majbaxter

Contributors KEC and $\mathrm{MAB}$ conceived the initial concept and designed the study, collected, verified, analysed and interpreted data, and drafted manuscript text. RDP verified and interpreted data. All authors helped write the manuscript and all approved the final manuscript.

Funding $\mathrm{MAB}$ is a Clinical Academic Fellow funded by the Scottish Chief Scientist Office.

Disclaimer All views expressed in the submitted article are the views of the authors and not an official position of our institution.

Competing interests RDP has undertaken speaking, consulting and advisory roles for Eli Lilly, BMS, Pfizer, Sanofi, Servier; and received research funding (not related to the work in this manuscript) from Astra Zeneca, Roche, MSD, Merck Serrano, Eli Lilly, Five Prime Therapeutics, Clovis, Boston Biomedical and Janssen.

Patient consent for publication Not required.

Ethics approval The study was performed with prospective authorisation from the local Caldicott guardian according to their requirements.

Provenance and peer review Not commissioned; externally peer reviewed.

Open access This is an open access article distributed in accordance with the Creative Commons Attribution Non Commercial (CC BY-NC 4.0) license, which permits others to distribute, remix, adapt, build upon this work noncommercially, and license their derivative works on different terms, provided the original work is properly cited, appropriate credit is given, any changes made indicated, and the use is noncommercial. See: http://creativecommons.org/licenses/by-nc/4. $0 /$.

ORCID iD

Mark A Baxter http://orcid.org/0000-0002-5773-8650

\section{REFERENCES}

1 Edgren G, Adami H-O, Weiderpass E, et al. A global assessment of the oesophageal adenocarcinoma epidemic. Gut 2013;62:1406-14.

2 UK, C.R. Oesophageal cancer, 2020. Available: https://www. cancerresearchuk.org/health-professional/cancer-statistics/ statistics-by-cancer-type/oesophageal-cancer/incidence\# heading-Zero

3 Merchant SJ, Brogly SB, Booth CM, et al. Palliative care and symptom burden in the last year of life: a population-based study of patients with gastrointestinal cancer. Ann Surg Oncol 2019;26:2336-45.

4 Cunningham D, Starling N, Rao S, et al. Capecitabine and oxaliplatin for advanced esophagogastric cancer. N Engl J Med 2008;358:36-46.

5 Waddell T, Chau I, Cunningham D, et al. Epirubicin, oxaliplatin, and capecitabine with or without panitumumab for patients with previously untreated advanced oesophagogastric cancer (REAL3): a randomised, open-label phase 3 trial. Lancet Oncol 2013;14:481-9.

6 Hall PS, Swinson D, Waters JS, et al. Optimizing chemotherapy for frail and elderly patients (PTS) with advanced gastroesophageal cancer (aGOAC): the Go2 phase III trial. JCO 2019;37:4006

7 Murad AM, Santiago FF, Petroianu A, et al. Modified therapy with 5-fluorouracil, doxorubicin, and methotrexate in advanced gastric cancer. Cancer 1993;72:37-41.

8 Pyrhönen S, Kuitunen T, Nyandoto P, et al. Randomised comparison of fluorouracil, epidoxorubicin and methotrexate (FEMTX) plus supportive care with supportive care alone 
in patients with non-resectable gastric cancer. Br J Cancer 1995;71:587-91.

9 Scheithauer W KG, Zeh B, et al. Palliative chemotherapy vs supportive care in patientes with metastatic gastric cancer: a randomized trial., in second International Conference on biology, prevention and treatment of Gi malignancy, Koln, Germany, 1995: 68.

10 Swinson D, Hingorani M, Stokes Z, et al. Best supportive care (BSC) with or without low-dose chemotherapy (chemo) in frail elderly patients with advanced gastroesophageal cancer (aGOAC): the uncertain randomization of the Go2 phase III trial. JCO 2019;37:4051.

11 Mills S, Buchanan D, Guthrie B, et al. Factors affecting use of unscheduled care for people with advanced cancer: a retrospective cohort study in Scotland. Br J Gen Pract 2019;69:e860-8.

12 Li D, Li D, Sun C-L. Patient-defined goals and preferences among older adults with cancer starting chemotherapy (CT). JCO 2018;36:10009.

13 CRUK. Improving cancer treatment and care for an ageing population, 2018.

14 ISD. Cancer statistics, 2019. Available: https://www. isdscotland.org/Health-Topics/Cancer/Cancer-Statistics/

15 Wagner AD, Syn NL, Moehler M, et al. Chemotherapy for advanced gastric cancer. Cochrane Database Syst Rev 2017;8:Cd004064. 